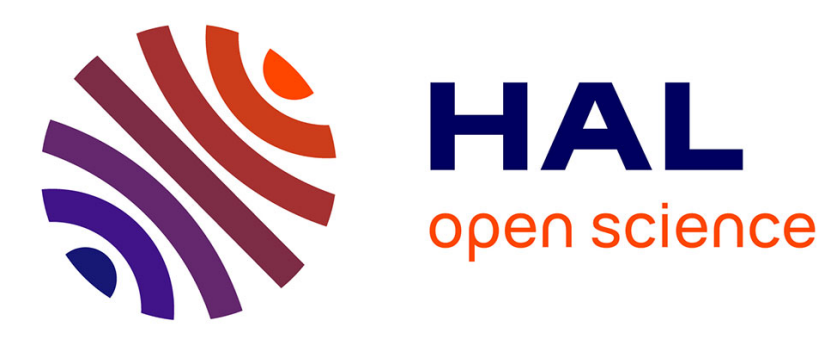

\title{
Distance-dependent Hückel-type model for the study of sodium clusters
}

Romuald Poteau, Fernand Spiegelmann

\section{To cite this version:}

Romuald Poteau, Fernand Spiegelmann. Distance-dependent Hückel-type model for the study of sodium clusters. Physical Review B: Condensed Matter and Materials Physics (1998-2015), 1992, 45 (4), pp.1878-1888. 10.1103/PhysRevB.45.1878 . hal-01755301

\section{HAL Id: hal-01755301 \\ https://hal.science/hal-01755301}

Submitted on 16 May 2019

HAL is a multi-disciplinary open access archive for the deposit and dissemination of scientific research documents, whether they are published or not. The documents may come from teaching and research institutions in France or abroad, or from public or private research centers.
L'archive ouverte pluridisciplinaire HAL, est destinée au dépôt et à la diffusion de documents scientifiques de niveau recherche, publiés ou non, émanant des établissements d'enseignement et de recherche français ou étrangers, des laboratoires publics ou privés. 


\title{
Distance-dependent Hückel-type model for the study of sodium clusters
}

\author{
Romuald Poteau and Fernand Spiegelmann \\ Laboratoire de Physique Quantique, Université Paul Sabatier, 118 Route de Narbonne, 31062 Toulouse CEDEX, France
}

(Received 3 June 1991; revised manuscript received 30 September 1991)

\begin{abstract}
A distance-dependent extension of the Hückel model is proposed and applied to sodium clusters. It consists primarily of a two-band monoelectronic formulation expressed in an $s+p$ basis set. The $s+p$ Hamiltonian is reduced into an $s$-only Hamiltonian by means of quasidegenerate perturbation theory, with the $p$ band treated perturbatively. The parametrization is taken from accurate calculations of $\mathrm{Na}_{2}$ and $\mathrm{Na}_{4}$. This formulation allows a very quick determination of the potential-energy surfaces, and the use of the Monte Carlo simulated-annealing technique for determining the stable isomers of clusters. For the smallest clusters $\left(\mathrm{Na}_{3}-\mathrm{Na}_{8}\right)$, the model provides stabilities and geometries in very good agreement with previous studies involving more sophisticated calculations ( $a b$ initio configuration-interaction or density-functional theory). Optimization results without constraint are also presented for clusters in the range $\mathrm{Na}_{9}-\mathrm{Na}_{19}$. Larger clusters in the range $\mathrm{Na}_{55}-\mathrm{Na}_{561}$ are examined with restricted symmetry constraints (icosahedra, cuboctahedra, and cubic clusters). Beyond $n=147$, the cuboctahedral structure is preferred.
\end{abstract}

\section{INTRODUCTION}

As prototype candidates for studying transition from the atom to the metal, alkali-metal clusters have in recent years attracted increasing interest. Unfortunately, due to their metallic nature, which is related to the delocalized character of the wave function, their stability and geometrical properties cannot be studied using oversimplified energy functions such as two- or three-body potentials or even diatom-in-molecules schemes. ${ }^{1}$ Among various treatments, one finds the jellium model or shell mod$\mathrm{el}^{2-10} a b$ initio calculations based on configuration interaction $(\mathrm{CI}),{ }^{11-16}$ or on density-functional theory, ${ }^{17-20}$ with or without the use of effective core pseudopotentials, and recently the topological Hückel model, ${ }^{21-23}$ which was shown to yield results in fair agreement with more accurate calculations.

The spherical jellium model was successful in predicting the stabilities of alkali-metal clusters, assuming almost complete screening of the nuclei and providing a cluster orbital picture which corresponds to a kind of superatom one, yielding spherical symmetries and rules for shell closing which explains the magic numbers. ${ }^{2}$ Spheroidal or ellipsoidal extensions ${ }^{4,7-9}$ of the jellium model were derived in order to study deviations from sphericity and were used to provide general shapes of clusters as oblate or prolate ellipsoids, showing also that within the jellium model, special stability at shell closing is associated with vanishing distortion from sphericity. However, the jellium model may present artifacts such as the metastable character of some clusters ${ }^{6,10}$ with respect to dissociation. The agreement with experiment in values for the dissociation energies or ionization potentials falls short of being quantitative. Moreover, since the nuclei do not appear explicitly, a description of the dynamics of fragmentation or clustering is apparently inaccessible to such a model, as is the study of crystallization properties.

On the other hand, $a b$ initio calculations ${ }^{11-16}$ were fairly successful in determining the geometries and stabil- ities of neutral and ionized alkali-metal clusters, as well as other properties such as ionization potentials and fragmentation energetics, in general good agreement with experimental results obtained in beams. The reliability of such $a b$ initio calculations was also recently confirmed by the study of excited states. ${ }^{24-26}$ Reliable $a b$ initio quantum-chemistry-type calculations require a substantial computational effort. The geometry optimization is particularly tedious since the potential-energy surfaces are rather flat and numerous isomers are in competition and often occur in near degeneracy. Gradient procedures have generally been applied only under the self-consistent field (SCF) approximation, and CI optimization is generally not exhaustive (optimization of a scaling factor for the SCF best structures). ${ }^{11-14}$ Thus only relatively small clusters have been studied and optimization is only partial or not fully reliable for $n \geq 10$. From another point of view, it is uncertain that properties observed in supersonic beams are directly and exclusively related to the description of geometrically static clusters. Besides, the use of $a b$ initio CI potential-energy surfaces (PES) in molecular dynamics or Monte Carlo simulation carries a prohibitive computational cost. A similar situation prevails for dynamic studies concerning fragmentation or clustering, except perhaps for three- or four-atom clusters.

An alternative $a b$ initio solution was provided by the density-functional approach ${ }^{17-20}$ ( DFT), which obtained results in general agreement with CI calculations, especially in the case of sodium clusters, yielding smaller interatomic distances, and probably overestimates of binding energies. Due to its efficiency, the DFT energy may be coupled to molecular-dynamics approaches such as the Car-Parinello method which was extensively used on metal and semiconductor clusters. ${ }^{27-30}$ However, few attempts have been done to date to use this method for the studying of fragmentation and clustering. Although much quicker than CI, DFT requires the computation of matrix elements in a given basis set, and the solution of a SCF problem which is replaced by a coupled-equation 
problem in the Car-Parinello formulation. ${ }^{27}$

Quite recently, some authors provided results using the simplest possible quantum-mechanical model, i.e., the Hückel topological model, ${ }^{21-23}$ the orbitals of which are expanded on $s$ atomic orbitals only. The search of the best structures consists in sorting a finite number of discrete topological structures. The results were found to be in surprising agreement with more sophisticated calculations. Although dealing with discrete topological structures, the sorting may turn to be difficult beyond ten atoms, since the exploration of discrete topological structures cannot be exhaustive, and the effort to calculate optimal graphs quickly becomes burdensome. The success of this latter model may appear paradoxical, since the only physics involved in it is delocalization (through the transfer integral $t$ ) without any explicit account of correlation, whereas $a b$ initio calculations show that large basis sets and extensive CI's are required in order to obtain reliable results; in other words, correlation effects are strong and cannot be neglected. This paradox was resolved in a recent work ${ }^{31,32}$ expressed in a valence-bond formulation where it was shown that the role of large basis sets in alkali-metal clusters was essentially to correlate and lower the ionic valence-bond forms, increasing thus the $t / U$ ratio of the Hubbard model, ${ }^{33}$ thus bringing those systems closer to the weakly correlated case (hypothesis of tight-binding models in solid-state physics). Although providing a very quick quantum-mechanical calculation of the energy, the topological Hückel model has two drawbacks.

(i) It provides only topologies and cannot be used for studying dynamical properties.

(ii) $p$ orbitals are not considered in the model, while they were shown to play some role even in small clusters. $^{11}$

The scope of the present paper is to propose a distance-dependent formulation of a Hückel-type model which allows for the obtention and optimization of real geometries, thus providing an efficient and realistic way to describe the potential-energy surfaces of alkali-metal clusters. Such a model is combined with gradient or Monte Carlo search for the optimal geometries of sodium clusters which are investigated in the range $n=2-14$ and compared with other results when available. The model, which takes into account $p$ orbitals in an effective way is introduced in Sec. II. Section III provides the results for $n=2-9$ and presents a systematic comparison with other existing data. Results in the range $n=10-14$, for which very few $a b$ initio type calculations have been published, are also given in Sec. III, as well as partial results concerning small clusters with $n \geq 15$. Section IV examines the stability and fragmentation properties that can be deduced from the present work. Finally, larger clusters of a few hundred atoms are investigated in Sec. V, and extrapolation to the bulk is considered.

\section{MODEL AND GEOMETRY OPTIMIZATION TECHNIQUES}

The traditional extended Hückel theory ${ }^{34}$ is expressed in a nonorthogonal basis set of atomic functions, and the geometry dependence is introduced through the overlap integrals. In the present work, the supposed basis set will be implicitly defined as composed of orthogonalized atomic functions. Alternatively to the previously mentioned topological Hückel model, ${ }^{21}$ we begin by considering a one-electron Hamiltonian for sodium clusters which is expressed in a space-fixed basis composed of one $s_{i}$ shell and one $p_{i}$ shell [including three $p$ functions $\left(\left\{p_{i}^{\mu}\right\}, \mu=x, y, z\right)$ per atom $\left.i\right]$. Thus in this formulation, the dimension of the matrix to be diagonalized is $4 n, n$ being the size of the cluster. The matrix elements are defined as follows.

(i) The diagonal elements $h_{i i}^{\alpha \alpha}$ are sums of two-body orbital repulsions $\rho_{i j}^{\alpha \gamma}$

$$
\begin{aligned}
& h_{i i}^{\alpha \alpha}=\sum_{j \neq i}^{\text {atoms }} \sum_{\gamma} \rho_{i j}^{\alpha \gamma}, \\
& \alpha, \gamma \in\left\{s, p_{x}, p_{y}, p_{z}\right\}
\end{aligned}
$$

the two-body terms $\rho_{i j}^{\alpha \gamma}$, which are functions of the interatomic distances, also depend on the nature and the orientations of the orbitals $\alpha$ and $\gamma$ on both sites (for $p$ orbitals). The scope of those diagonal terms is to introduce an interatomic repulsion, which is generally lacking or poorly represented in the ordinary and extended Hückel model.

(ii) The off-diagonal elements are assumed to follow the usual overlap scheme and the one-center $h_{i i}^{\alpha \gamma}(\alpha \neq \gamma)$ elements are supposed to be zero. The two-center integrals $h_{i j}^{\alpha \gamma}(i \neq j)$, also considered hereafter as functions of the distances, are taken as distance-dependent transfer integrals $t_{i j}^{\alpha \gamma}$ as defined in the Hückel model.

We now introduce a simplification of the above $4 n \times 4 n$ model, by projecting the problem into the basis set of $s$ only orbitals treating the $p$ orbitals by means of quasidegenerate-perturbation theory (QDPT) and the theory of effective Hamiltonians. ${ }^{35}$ In the $s$-only basis set, the matrix elements $\widetilde{h}_{i j}^{s s}$ perturbed up to the second order, can then be expressed as

$$
\begin{aligned}
& \tilde{h}_{i i}^{s s}=h_{i i}^{s s}+\sum_{k \neq i}^{\text {atoms }} \sum_{\alpha} \frac{\left(h_{i k}^{s \alpha}\right)^{2}}{\Delta E_{i k}^{s \alpha}}, \alpha \in\left\{p_{x}, p_{y}, p_{z}\right\}, \\
& \tilde{h}_{i j}^{s s}=h_{i j}^{s s}+\sum_{\substack{k \neq i, \alpha \\
k \neq j}}^{\text {atoms }} \sum_{\substack{\alpha \\
k \neq}} \frac{h_{k j}^{s \alpha} h_{k j}^{\alpha s}}{\Delta E_{i k}^{s \alpha}} .
\end{aligned}
$$

Moreover, in $h_{i i}^{s s}$, the $s-p$ repulsion integrals $\rho_{i j}^{s p}$ are neglected, with only the isotropic $\rho_{i j}^{s s}$ repulsions retained and $\Delta E_{i k}^{s \alpha}$ is approximated as the asymptotic transition $\Delta E_{s p}=0.0773$ a.u. for sodium. This means that the $s$ basis set is assumed to account for the main physical effects in alkali-metal clusters (in line with the relative success of the topological Hückel model) and is chosen as the model space of the QDPT theory. Nevertheless, $p$ orbitals, though not treated variationally, are introduced via perturbation terms. In particular, the direct interactions between the two bands are explicitly considered in the perturbation, with the interband separation $\Delta E_{s p}$ kept constant. Thus, the repulsion in the $s$ band is lowered due to mixing with the $p$ band, yielding an effective diagonal term $\widetilde{h}_{i i}^{s s}$ in the Hamiltonian. The intersite transfer 
$\tilde{h}_{i j}^{s s}$ integral is also modified due to interactions with $p$ orbitals located on neighboring sites. An interesting property of this perturbative formulation, especially for the off-diagonal terms, is that it introduces explicitly threebody interactions in the $\widetilde{h}_{s s}^{i j}$ effective transfer integrals, beyond the two-body terms defining the transfer in the $s$ band. Those three-body transfer terms are, in general, smaller than two-body transfer terms. However, they may happen to be the only nonvanishing terms when two centers $i$ and $j$ are widely separated but connected via a third center $k$ lying close enough to both of them. Thus those three-body terms are likely to introduce some long-range interactions in the Hamiltonian.

From a practical point of view, there are two advantages in the final formulation. First, it reduces the size of the matrix to be diagonalized, which is now equal to the number of atoms. Second, it reduces the number of integrals to be parametrized in the model, since only the $s-s$ integrals $\rho_{i j}^{s s}$, and $t_{i j}^{s s}$ and the $s-p$ integrals $t_{i j}^{s \alpha}$ are kept.

The total energy is computed as a sum of eigenvalues corresponding to one-electron eigenfunctions.

$\tilde{h}\left|\varphi_{k}\right\rangle=\varepsilon_{k}\left|\varphi_{k}\right\rangle,\left|\varphi_{k}\right\rangle=\sum_{i} c_{k i}\left|s_{i}\right\rangle, \quad E=\sum_{k \in \text { occ }} n_{k} \varepsilon_{k}$.

The integrals $\rho_{i j}^{s s}$ and $t_{i j}^{s s}$ are isotropic, while the integrals $t_{i j}^{s \alpha}$ depend on the nature of the $\alpha$ space-fixed $p$-type function and its orientation with respect to the internuclear axis $i j$. In order to provide a simple trigonometric formulation, $\alpha$ is decomposed into two terms, respectively, parallel and perpendicular to the internuclear axis:

$$
t_{i j}^{s \alpha}=\left\langle s_{i}|\tilde{h}| p_{j}^{\alpha}\right\rangle=\left\langle s_{i}|\tilde{h}| p_{j}^{\sigma}\right\rangle+\left\langle s_{i}|\tilde{h}| p_{j}^{\pi}\right\rangle .
$$

If we again follow the usual overlap scheme for the cancellation of matrix elements, we are left only with the first term which reads

$$
t_{i j}^{s \alpha}=\cos \theta_{i j}^{\alpha} t_{i j}^{s \sigma},
$$

where $t_{i j}^{s \sigma}$ is the transfer integral between the orbital $s_{i}$ and a $p_{j}^{\sigma}$ orbital along the local axis $i j$. $\theta_{i j}^{\alpha}$ is the angle between the local axis $i j$ and the axis defining the spacefixed initial orbital $p_{j}^{\alpha}$.

In order to determine the three integrals $\rho_{i j}^{s s}, t_{i j}^{s s}$, and $t_{i j}^{s \sigma}$, a least-squares fit was achieved in order to reproduce at any distance the potential-energy curve of the $X^{1} \Sigma_{g}^{+}$ ground state and the ${ }^{3} \Sigma_{u}^{+}$first excited state determined by Jeung ${ }^{36}\left(R_{e}=5.8 a_{0}\right.$, and $D_{e}=0.74 \mathrm{eV}$, in agreement with experiment ${ }^{37}$ ), as well as the dissociation energy $\left(0.41 \mathrm{eV}\right.$ into $\left.\mathrm{Na}_{2}+\mathrm{Na}_{2}\right)$ of the optimized ${ }^{1} A_{g}$ rhombus, which is the most stable structure of $\mathrm{Na}_{4}$ taken from the recent work of Bonačić-Koutecký, Fantucci, and Koutecký, ${ }^{25}$ imposing moreover a relation between $t_{i j}^{s s}$ and $t_{i j}^{s \sigma}$ following the usual overlap scheme,

$$
\frac{t_{i j}^{s s}}{t_{i j}^{s \sigma}}=\frac{S_{i j}^{s s}}{S_{i j}^{s \sigma}},
$$

where $S_{i j}^{s s}$ and $S_{i j}^{s \sigma}$ are, respectively, the $s$-s and $s-p_{\sigma}$ overlap integrals. $\mathrm{Na}_{4}$ is the first cluster exhibiting some nonvanishing $s-p$ mixing allowing a significant influence of the $t_{i j}^{s \sigma}$ integral. The $\rho^{s s}, t^{s s}$, and $t^{s \sigma}$ functions are listed in Table I. Finally, the algorithm uses a point grid of integrals values interpolated through cubic spline functions at any required distance, which is more efficient than computing exponential-type analytical expressions.

The present method is in the spirit of the tight-binding (TB) formulation by Chadi and co-workers ${ }^{38}$ in which a semiempirical distance dependence was added to the tight-binding term in order to parametrize the ion-ion interaction energy. The parametrization of Chadi has been widely used in the study of silicon solids or surfaces ${ }^{39}$ and also in the case of silicon clusters, ${ }^{40}$ eventually including a Hubbard-type term to penalize two-electron on-site repulsion. Recently, more sophisticated extensions of the TB model were proposed in order to introduce electrostatic terms. One may quote the tight-binding bond (TBB) model of Sutton et al., ${ }^{41}$ and also the selfconsistent tight-binding (SCTB) method proposed by Majeski and $\operatorname{Vog}^{42}$ and developed by Kohyama et al. ${ }^{43}$ The primary contribution of the present method is the development of a perturbative treatment for the $3 p$ shell, which accounts for the hybridization effects in cases where $p$ orbitals are not occupied in the atoms. This perturbative treatment still provides a rather efficient algorithm and can be used in wide-range optimization and simulation requiring numerous calculations of the energy function. The parametrization of the matrix elements relies on $a b$ initio results for the smaller clusters and not on semiempirical formulas.

In order to find the optimized geometry of clusters, two procedures are used hereafter. The first one is gradient optimization without symmetry constraints in the framework of the Hellmann-Feynman theorem. We have moreover neglected the derivatives of the $t^{s \sigma}$ integrals, which are only involved in the perturbation. The energy gradient with respect to the coordinates $q_{i \mu}$ can be expressed

$$
\begin{aligned}
\frac{\partial E}{\partial q_{i \mu}}=\sum_{k \in \mathrm{occ}} n_{k} \frac{\partial \varepsilon_{k}}{\partial q_{i \mu}} & =\sum_{k \in \text { occ }} n_{k}\left\langle\varphi_{k}\left|\frac{\partial \tilde{h}}{\partial q_{i \mu}}\right| \varphi_{k}\right\rangle \\
& =\sum_{k \in \text { occ }} n_{k} \sum_{l} \sum_{m} c_{k m} c_{k l} \frac{\partial \tilde{h}_{l m}}{\partial q_{i \mu}} ;
\end{aligned}
$$

the $\partial \tilde{h}_{l m} / \partial q_{i \mu}$ derivatives are readily obtained through cubic spline coefficients interpolation of the integrals cor-

TABLE I. Values (in eV) of the $t^{s s}, \rho^{s s}$, and $t^{s \sigma}$ parameters as functions of the internuclear distance.

\begin{tabular}{lclc}
\hline \hline$R$ & $t^{s s}$ & \multicolumn{1}{c}{$\rho^{s s}$} & $t^{s \sigma}$ \\
\hline 4 & -0.026747 & 0.183205 & 0.005932 \\
5 & -0.340642 & 0.048189 & 0.164076 \\
5.5 & -0.373267 & 0.024679 & 0.186633 \\
6 & -0.367417 & 0.012217 & 0.215204 \\
6.5 & -0.336724 & 0.005768 & 0.215394 \\
7 & -0.292317 & 0.002558 & 0.203204 \\
7.5 & -0.242631 & 0.000299 & 0.182579 \\
8 & -0.193626 & 0.000101 & 0.157192 \\
9 & -0.111534 & 0.000082 & 0.104541 \\
12 & -0.013659 & 0.0 & 0.018884 \\
15 & 0.0 & 0.0 & 0.0 \\
\hline \hline
\end{tabular}


responding to the matrix elements.

The second technique is the Monte Carlo simulatedannealing procedure ${ }^{44}$ which, in principle, provides a nonlocal (global) optimization algorithm when the initial temperature is high, but which can also be used as a local algorithm when the initial temperature is low. Both gradient and low-energy simulated annealing can be used simultaneously as mutual checks for real minima.

For Monte Carlo optimization, the Metropolis ${ }^{45}$ algorithm is used and the conditions of the simulated annealing process are the following.

(i) Start the $T=600 \mathrm{~K}$ with a given arbitrary initial geometry (the melting temperature of solid sodium being $371 \mathrm{~K})$.

(ii) Imposing a linear cooling rate of $-33 \mathrm{~K} / \mathrm{step}$.

(iii) For each cooling step, the number of random configurations is proportional to the number of atoms with a factor of 250 .

(iv) With regard to moves in configurations space $\left\{q_{i \mu}\right\}$, the coordinate $q_{i \mu}$ is taken to be random while its variation is kept constant at $\delta q_{i \mu}=0.25$ bohr for $T \geq 1.5$ $\mathrm{K}$, and at $\delta q_{i \mu}=0.01 \mathrm{bohr}$ for $T \leq 1.5 \mathrm{~K}$. The sign of the displacement is also random.

(v) The process is assumed to converge when at some temperature step no further displacement is validated by the Monte Carlo process. In the case of local optimization, the initial temperature is chosen to be $<10 \mathrm{~K}$.

\section{RESULTS AND DISCUSSIONS FOR CLUSTERS $n=2-14$}

The equilibrium constants for the dimer yielded by the present model are $R_{e}=5.9 a_{0}$ and $D_{e}=0.72 \mathrm{eV}$, differing only slightly from the initial inputs. This difference is due to deviations in the least-squares fit. In dealing with cluster energetics, it is important to give a fairly correct description of the dimer. Indeed, it was shown theoretically ${ }^{46,47}$ for neutrals and ions and experimentally ${ }^{47}$ in the case of ions that unimolecular dissociation of alkali-metal clusters is governed by only two dominant dissociation channels, respectively, the evaporation of a monomer and the evaporation of a dimer. Thus, the determination of the lowest dissociation channel requires some accuracy and consistency for the dimer dissociation energy. This is not always true in $a b$ initio CI calculations for clusters which are generally performed in rather small basis sets, thus underestimating the dimer stability, in particular, nor in DFT calculations that alternatively tend to overestimate this stability.

MCSA (Monte Carlo simulated annealing) was used in order to determine the most stable structures of clusters from $\mathrm{Na}_{3}$ to $\mathrm{Na}_{9}$, which are depicted in Fig. $1\left(\mathrm{Na}_{2}-\mathrm{Na}_{6}\right)$ and Fig. $2\left(\mathrm{Na}_{7}-\mathrm{Na}_{9}\right)$. Other isomers on the potentialenergy surfaces are also shown. $U p$ to $\mathrm{Na}_{8}$, the most stable structures are in agreement with the $a b$ initio calculations of Bonačić-Koutecký, Fantucci, and Koutecký ${ }^{14}\left(\mathrm{Na}_{2}-\mathrm{Na}_{9}\right)$ and Spiegelmann and Pavolini ${ }^{16}$ $\left(\mathrm{Na}_{2}-\mathrm{Na}_{6}\right)$, including shape, spatial symmetry of the states (excluding spin which is neglected here) and bonds. It is interesting to note that contrary to results of the topological Hückel model, the structure of the trimer is ob-

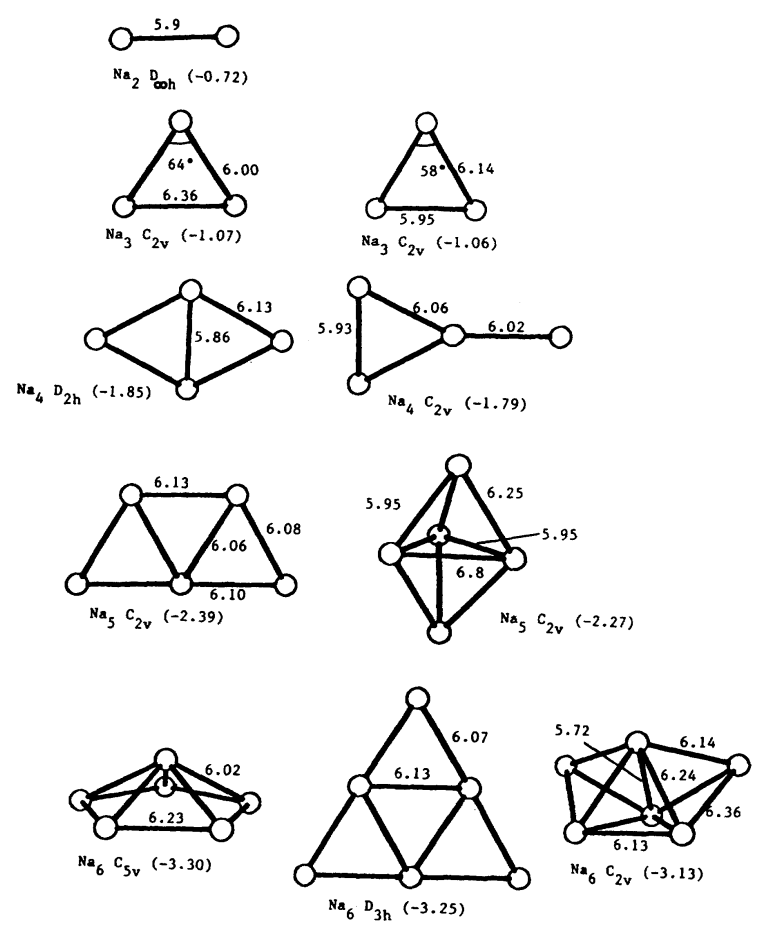

FIG. 1. Geometries and total energies (in eV) of $\mathrm{Na}_{2}-\mathrm{Na}_{6}$ clusters.

tained in the present model as an isosceles triangle with an apex angle of $64^{\circ}$, that is to say that the Jahn-Teller distortion obtained, which is responsible for the deviation from the equilateral triangle, is in the right direction, although it is known that the energy differences between the two isosceles shapes $\left(\theta>60^{\circ}\right.$ and $\left.\theta<60^{\circ}\right)$ are very small in alkali-metal trimers. ${ }^{48-54}$ For the tetramer, the sides of the rhombus are larger than the small diagonal,
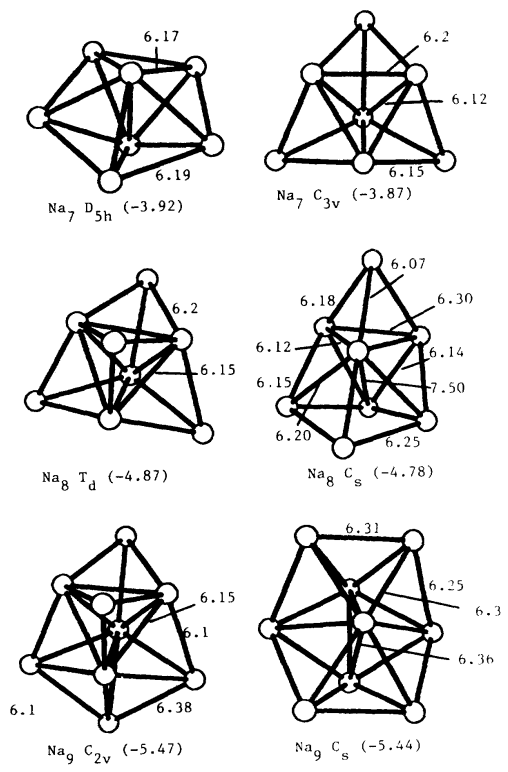

FIG. 2. Geometries and total energies (in $\mathrm{eV}$ ) of $\mathrm{Na}_{7}-\mathrm{Na}_{9}$ clusters. 
in agreement with the results of Bonačić-Koutecký, Fantucci, and Koutecký ${ }^{14}$ and of Martins, Car, and Buttet $^{18,19}$ while all distances were found equal in the work of Spiegelmann and Pavolini. ${ }^{16}$ Another structure with $\mathrm{T}$ shape is found at higher energy. As concerns the pentamer ground state, which is planar, all bond distances are found almost equal, in agreement with previous authors. The triangular bipyramid is found as an isomer. For $\mathrm{Na}_{6}$, the two isomers $\left(C_{5 v}\right.$ pyramid and $D_{3 h}$ bitriangle) are found to be quasidegenerate, although the $C_{5 v}$ structure is the ground state. The distortion from planar shape is however slightly accentuated with our model for the $C_{5 v}$ pyramid. A third isomer (bicapped tetrahedron) is found $0.17 \mathrm{eV}$ higher. As concerns the $\mathrm{Na}_{7}$ ground state $\left(D_{5 h}\right.$ bipyramid), its parameters are again consistent with the work of Bonačić-Koutecký, Fantucci, and Koutecký ${ }^{14}$ and Martins, Car, and Buttet, ${ }^{18,19}$ with an axis length larger than the pentagon sides. The two atoms on the axis are nevertheless bonded, contrary to what was obtained in the Hückel topological model. Two higher structures are found for $\mathrm{Na}_{7}$, the first one being the tricapped tetrahedron, the second one being obtained by rotating symmetrically two of the capped atoms around the tetrahedron edges. The ground state of $\mathrm{Na}_{8}$ is found to be the $T_{d}$ fully capped tetrahedron, as in the work of Bonačić-Koutecký, Fantucci, and Koutecký, whereas the DFT calculations provided a structure with lower symmetry $D_{2 h} \cdot{ }^{18,19}$ Neither the $D_{2 h}$ structure nor the $D_{4 d}$ square antiprism are found to be minima on the potential surface, and this is consistent with $a b$ initio CI calculations. ${ }^{14}$ We obtain however a second minimum with $C_{s}$ symmetry which lies $0.09 \mathrm{eV}$ above the ground state. In the case of $\mathrm{Na}_{9}$, two isomers are found very close in energy, namely, the singly capped octamer with $C_{2 v}$ symmetry $\left(\mathrm{Na}_{8}\right.$ plus one capping atom) and the doubly capped pentagonal bipyramid ( $\mathrm{Na}_{7}$ plus two capping atoms). However, in the present work, the $C_{2 v}$ structure is slightly lower, while Bonačić-Koutecký, Fantucci, and Koutecký provide the $C_{s}$ geometry as the ground state. One may remark that the same authors ${ }^{11,15}$ find the $C_{2 v}$ structure to be the lowest for $\mathrm{Li}_{9}$.

The binding energies per atom (taking as zero energy the energy of one atom)

$$
e(n)=[n E(1)-E(n)] / n=-E(n) / n,
$$

are reported in Table II for the ground-state structures and compared to previous results. As concerns the smallest clusters, accurate $a b$ initio calculations in extensive basis sets are available. Our binding energy for the trimer $(8.19 \mathrm{kcal})$ agree fairly well with values taken from the recent $a b$ initio calculation of Jeung, ${ }^{54} 7.94$ kcal, while an experimental value of $8.20 \mathrm{kcal}$ can be deduced from the work of Hilpert ${ }^{55}$ and the dissociation energy of $\mathrm{Na}_{2}(0.74 \mathrm{eV})$. The binding energy of $\mathrm{Na}_{4}$ is, of course, in close agreement with the $a b$ initio result of BonačićKoutecký, Fantucci, and Koutecký ${ }^{25}$ which was used as input in the least-squares procedure. For larger clusters with $5 \leq n \leq 9$, the only results available are CI calculations performed in smaller basis sets, ${ }^{14}$ the DFT calculations of Martins, Buttet, and Car, the very recent DFT results of Röthlisberger and Andreoni ${ }^{29}$ using the CarParinello method, and results provided by the topological Hückel model. ${ }^{21,22}$ It can be seen in Fig. 3 that up to $n=8$, the binding energy per atom obtained in the present model follows the trend obtained in $a b$ initio calculations and also in the topological Hückel model. They are very close to the results of the calculations of Spiegelmann and Pavolini ${ }^{16}$ which only concern $n \leq 6$, and generally above the values of Bonačić-Koutecký, Fantucci, and Koutecký ${ }^{14}$ who used smaller basis sets and did not

TABLE II. Binding energies per atoms of $\mathrm{Na}_{n}$ clusters (in $\mathrm{kcal} / \mathrm{mol}$ ). Density-functional theory, Martin, Car, and Buttet (Refs. 18 and 19) [DFT(MCB)]; density-functional theory, Röthlisberger and Andreoni (Ref. 29) [DFT(RA)]; all electron CI, Bonačić-Koutecký, Fantucci, and Koutecký (Ref. 14) (AE-CI); effective core potential CI, Bonačić-Koutecký, Fantucci, and Koutecký (Ref. 14) [ECP-CI (BFK)]; Spiegelmann and Pavolini (Ref. 16) [ECP-CI (SP)]; topological Hückel Model, Wang et al. (Refs. 21 and 22) (HMO).

\begin{tabular}{|c|c|c|c|c|c|c|c|}
\hline$n$ & DFT(MCB) & DFT(RA) & AE-CI & $\mathrm{ECP}-\mathrm{CI}(\mathrm{BFK})$ & $\mathrm{ECP}-\mathrm{CI}(\mathrm{SP})$ & HMO & This work \\
\hline 2 & 10.34 & 9.65 & 6.25 & 6.25 & 8.07 & 8.73 & 8.276 \\
\hline 3 & 9.88 & 9.27 & 5.90 & 5.80 & 7.29 & 8.73 & 8.191 \\
\hline 4 & 14.02 & 12.36 & 8.82 & 7.96 & 10.16 & 11.19 & 10.603 \\
\hline 5 & 14.71 & 13.10 & 9.53 & 8.31 & 10.85 & 11.61 & 10.988 \\
\hline 6 & 16.78 & 15.32 & 10.87 & 9.77 & 12.62 & 13.64 & 12.636 \\
\hline 7 & 18.85 & 16.29 & 11.00 & 10.00 & & 13.86 & 12.873 \\
\hline 8 & 19.77 & 17.61 & 12.00 & 10.75 & & 15.46 & 14.000 \\
\hline 9 & & 16.98 & 10.75 & & & 15.19 & 13.977 \\
\hline 10 & & 17.65 & & & & 15.42 & 14.453 \\
\hline 11 & & & & & & 15.28 & 14.437 \\
\hline 12 & & & & & & 15.73 & 14.816 \\
\hline 13 & 19.77 & & & & & 16.13 & 15.023 \\
\hline 14 & & & & & & 16.48 & 15.323 \\
\hline 15 & & & & & & & 15.456 \\
\hline 16 & & & & & & & 15.758 \\
\hline 17 & & & & & & & 16.043 \\
\hline 19 & & & & & & & 16.439 \\
\hline
\end{tabular}


include the core-valence correlation effects. Both DFT binding energies are systematically larger than other calculated values, and also exhibit a quicker increase towards the bulk value especially in the calculation of Martins, Buttet and Car. ${ }^{19}$ One may notice the odd-even alternation in the stabilities for all calculations which was however not present in the DFT result of Martins, Buttet, and $\mathrm{Car}^{19}$ for $n \geq 6$. If one assumes that magic numbers directly reflect the stability properties, according to the prediction of the spherical jellium model, particular stabilities are expected at shell closings 2 and 8 , which are actually obtained in the present model (as in some other calculations), with drops for $n=3$ and 9. However, in our results, the drop at $n=9$ is less pronounced than in other calculations taking into account geometrical structure or in the spherical jellium model.

A further interesting property of the model is that not only the best structures are obtained, but also higher isomers are found at energies consistent with those obtained in $a b$ initio calculations. In particular in the trimer case, the ${ }^{2} A_{1}$ isosceles structure is very close to the ${ }^{2} B_{2}$ ground state, in good agreement with most theoretical calculations which yield a very flat surface along the pseudorotation path. For $\mathrm{Na}_{4}$, the $\mathrm{T}$-shape isomer is closer to the ground state $(0.058 \mathrm{eV})$ than in the work of BonačićKoutecký, Fantucci, and Koutecký ${ }^{14}(0.21 \mathrm{eV})$.

In the pentamer case, the $C_{2 v}$ bipyramid is $0.12 \mathrm{eV}$,

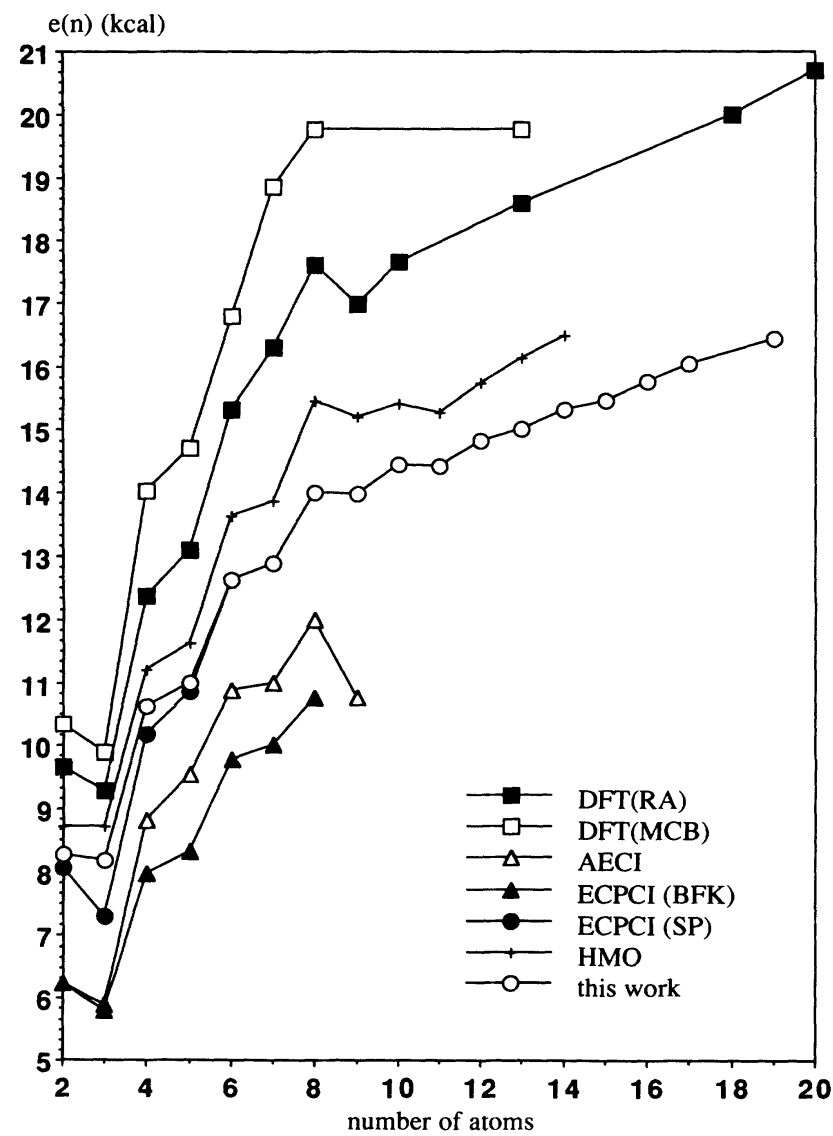

FIG. 3. Binding energies per atom for small sodium clusters. References and labeling are the same as in Table II. above the trapezoidal ground state $[0.22 \mathrm{eV}$ in the $\mathrm{AE}$ (where AE denotes all electron) work of BonačićKoutecký, Fantucci, and Kouteckýl ${ }^{14}$ ]. With regard to the two lowest quasidegenerate hexamers, the difference is found to be $0.046 \mathrm{eV}$, which can be compared to the 0.024-eV value of Spiegelmann and Pavolini ${ }^{16}$ and the 0.040-eV value of Bonačić-Koutecký, Fantucci, and Koutecký. ${ }^{14}$ One may thus infer that the present model is not only able to reproduce the optimized ground-state structures, but it also provides a rather fair representation of the potential-energy surfaces of small sodium clusters.

It should be stressed that the small clusters geometries determined in the present work are close to be homothetic to those calculated by Spiegelmann and Pavolini, or Bonačić-Koutecký, Fantucci, and Koutecký; ${ }^{14}$ the bond distances being generally smaller than those of the $a b$ initio calculations.

In the range $n=10-19$, the number of quasidegenerate isomers increases, and although we have used the same MCSA schedule, it cannot of course be ensured that all the lowest minima have been found, especially for $n \geq 15$. Apart from the results of the jellium model (in either version, spherical, ${ }^{2,3}$ spheroidal, ${ }^{8,9}$ or ellipsöidal ${ }^{7}$ ), only very few results are available in this range of sizes. Even in the topological Hückel model, optimization for $n \geq 10$ cannot be exhaustive because of the number of discrete structures to be considered. One should quote in the literature cases treated within the DFT formalism ${ }^{20}$ and the symmetry-based crystal-field estimations of Mingos ${ }^{56,57}$ for clusters corresponding to shell closings.

In the case of $\mathrm{Na}_{10}$, three structures (Fig. 4) are very close in energy. Two of them have $C_{4 v}$ symmetry, name-
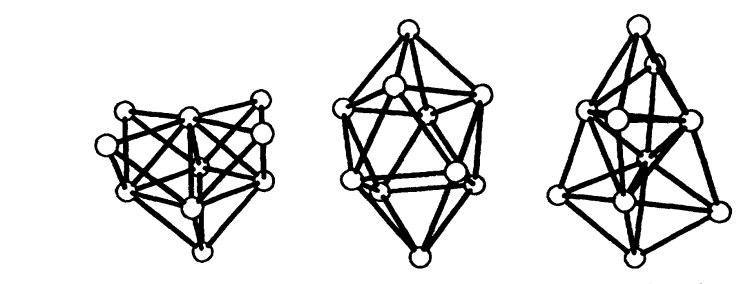

$$
\mathrm{Na}_{10} \mathrm{C}_{4 \mathrm{v}}(-6.29)
$$

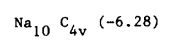

$\mathrm{Na}_{10} \mathrm{C}_{2 \mathrm{v}}(-6.25)$
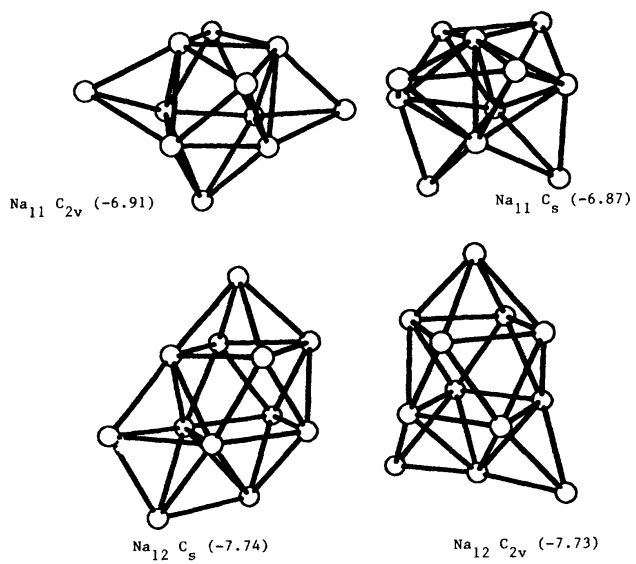

FIG. 4. Geometries and total energies (in $\mathrm{eV}$ ) of $\mathrm{Na}_{10}-\mathrm{Na}_{12}$ clusters. 
ly, a tetracapped square bipyramid (our actual ground state) and a bicapped square antiprism which was also obtained by Lindsay, Wang, and George. ${ }^{22}$ We obtain as third structure the $C_{2 v}$-bicapped octamer (it can be noted that this geometry is found in the $\mathrm{Li}_{10}$ case by BonačićKoutecký, Fantucci, and Koutecký. ${ }^{11,15}$ The most stable shape (Fig. 4) for $\mathrm{Na}_{11}$ is a fully capped trigonal prism as in the work of Lindsay, Wang, and George. ${ }^{22}$ The present structure is slightly distorted from $D_{3 h}$ symmetry due to the Jahn-Teller effect. An isomer is obtained at higher energy and corresponds to a tetracapped pentagonal bipyramid. Its geometry can, however, be viewed as a fully capped trigonal bipyramid, again distorted from $D_{3 h}$ symmetry due to Jahn-Teller degeneracy. With regard to $\mathrm{Na}_{12}$, two degenerate isomers (Fig. 4) are found; both correspond to a double capping of the second isomer of $\mathrm{Na}_{10}$ (i.e., the doubly capped square antiprism). In one case, the capping takes place on two opposed faces, while in the second case it takes place on two adjacent faces. Although this latter structure presents an extra bond, its energy is not significantly lowered.

The ground state of $\mathrm{Na}_{13}$ has $C_{1}$ symmetry and is an hexacapped pentagonal bipyramid (Fig. 5). Three higher isomers were obtained for $\mathrm{Na}_{13}$, none of them being an icosahedron, which is Jahn-Teller forbidden. The lowest isomer has $C_{2 v}$ symmetry and can be understood as consisting of three embedded pentagonal bipyramids. Two other isomers are very close. One with $C_{1}$ symmetry essentially consists of two embedded bipyramids with an extra connecting atom, while the second one $\left(C_{2 v}\right)$ can be obtained by capping with two atoms the second isomer of $\mathrm{Na}_{11}$. In the case of $\mathrm{Na}_{14}$, the ground state has $C_{s}$ sym-
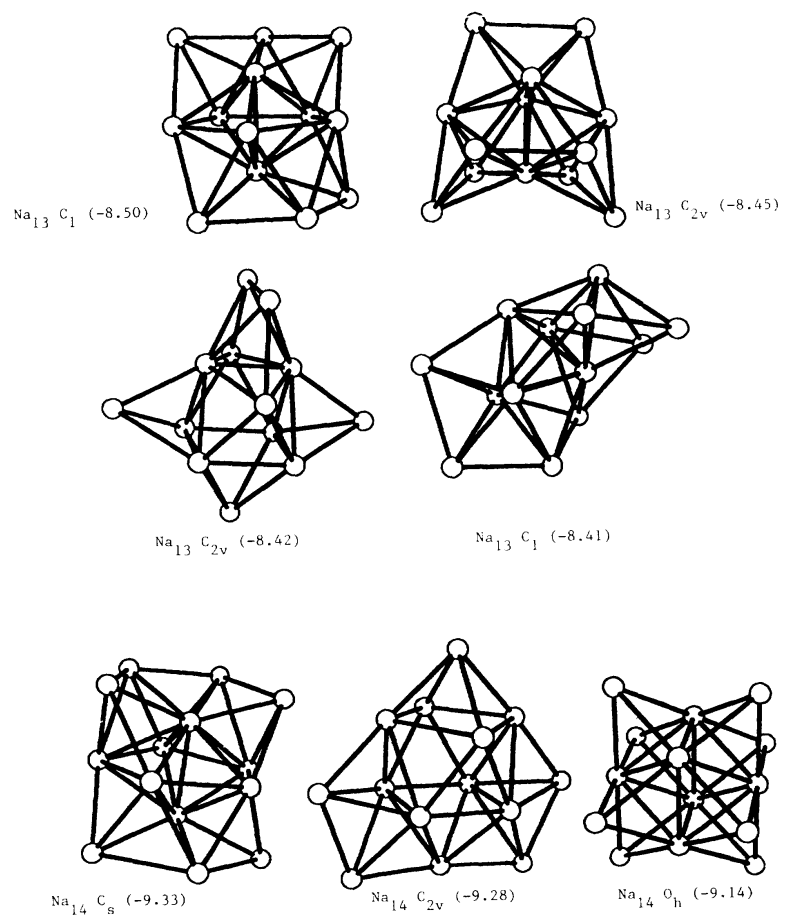

FIG. 5. Geometries and total energies (in eV) of $\mathrm{Na}_{13}$ and $\mathrm{Na}_{14}$ clusters. metry and is obtained by partially capping the faces of the pentagonal bipyramid with seven atoms (three above, four below). The second structure of $\mathrm{Na}_{14}$ has $C_{2 v}$ symmetry and is obtained by symmetrically capping the second structure of $\mathrm{Na}_{10}$ with four atoms. A third structure higher by $0.15 \mathrm{eV}$ is more symmetrical $\left(O_{h}\right)$ and corresponds to a piece of the bcc lattice. This isomer was found by Fantucci and Koutecký in the case of $\mathrm{Li}_{14}{ }^{15}$

Our investigation was less systematic beyond $\mathrm{Na}_{14}$. $\mathrm{Na}_{15}$ has $C_{s}$ symmetry and can be understood as an icosahedron capped with two contiguous atoms. $\mathrm{Na}_{16}$ also has $C_{s}$ symmetry and consists of an icosahedron capped by three contiguous atoms. $\mathrm{Na}_{17}$ has $C_{s}$ symmetry and consists of an icosahedron capped by a set of four atoms. $\mathrm{Na}_{19}$ has $D_{5 h}$ symmetry and is found to be the double icosahedron, also found in the case of $\mathrm{Li}_{19}$ (see Fig. 6). ${ }^{15}$ We note that $\mathrm{Na}_{17}$ can be obtained from $\mathrm{Na}_{19}$ by removing two atoms. Moreover, it should be stressed that the ground-state geometries found for $\mathrm{Na}_{9}, \mathrm{Na}_{10}$, and $\mathrm{Na}_{13}$ correspond to those found in the recent work of Röthlisberger and Andreoni. ${ }^{29}$

It is interesting to compare the shapes of the clusters obtained in the present work taking into account the positions of the nuclei with those obtained in the recent spheroidal $^{8,9}$ or ellipsoidal ${ }^{7}$ versions of the jellium model. Table III presents the ratios of the moments of inertia in the range $n=6-14$ as compared with those of the ellipsoidal jellium model ${ }^{7}$ (smaller clusters are planar). Of course $\mathrm{Na}_{8}$, with $T_{d}$ symmetry although not really spherical, has three equivalent axes. Clusters $\mathrm{Na}_{6}$ and $\mathrm{Na}$ are oblate, while clusters $\mathrm{Na}_{9}-\mathrm{Na}_{12}$ are prolate and $\mathrm{Na}_{13}$ and $\mathrm{Na}_{14}$ are again oblate. However, some discrepancies occur, the ground state of $\mathrm{Na}_{10}$ is oblate in our calculation, whereas the first isomer is actually prolate. Also sometimes, two axes are found equivalent in the jellium model, while they differ in our work.
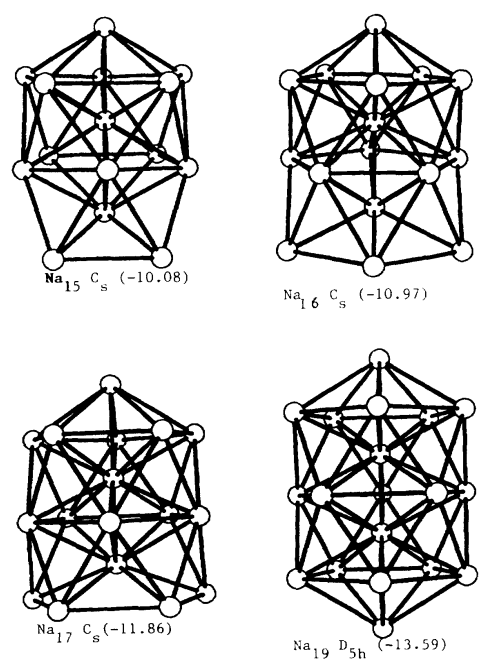

FIG. 6. Geometries and total energies (in $\mathrm{eV}$ ) of $\mathrm{Na}_{15}, \mathrm{Na}_{16}$, $\mathrm{Na}_{17}$, and $\mathrm{Na}_{19}$ clusters. 
TABLE III. Axial ratios of $\mathrm{Na}_{n}$ clusters. The present results are reported in the first row. The second one refers to the ellipsoidal jellium results of Selby et al. (Ref. 7). 10* corresponds to the second structure obtained for $n=10$, quasidegenerated with the ground state.

\begin{tabular}{llll}
\hline \hline$n$ & $x$ & $y$ & \multicolumn{1}{c}{$z$} \\
\hline 6 & 0.47 & 1.46 & 1.46 \\
& 0.711 & 1.186 & 1.186 \\
7 & 0.66 & 1.22 & 1.22 \\
& 0.875 & 1.069 & 1.069 \\
8 & 1 & 1 & 1 \\
& 1 & 1 & 1 \\
9 & 0.91 & 0.93 & 1.18 \\
& 0.914 & 0.914 & 1.196 \\
10 & 0.88 & 1.06 & 1.06 \\
$10^{*}$ & 0.88 & 0.88 & 1.28 \\
& 0.860 & 0.860 & 1.352 \\
11 & 0.89 & 0.91 & 1.24 \\
& 0.809 & 0.917 & 1.348 \\
12 & 0.74 & 1.00 & 1.36 \\
& 0.770 & 0.963 & 1.348 \\
13 & 0.69 & 1.08 & 1.34 \\
& 0.736 & 1.082 & 1.255 \\
14 & 0.72 & 1.10 & 1.26 \\
& 0.711 & 1.186 & 1.186 \\
\hline \hline
\end{tabular}

\section{STABILITY AND FRAGMENTATION PROPERTIES}

For larger clusters in the range $\mathrm{Na}_{10}-\mathrm{Na}_{19}$ the binding energy per atom is continuously increasing, with a less accentuated slope than in the range $\mathrm{Na}_{2}-\mathrm{Na}_{9}$. The oddeven alternation in the stabilities still exists but is significantly smoothed with respect to the smaller clusters.

We can use the present results in order to examine the unimolecular fragmentation properties of sodium cluster with respect to energy. It was shown for the smaller alkali-metal clusters, that two channels are generally predominant $^{11-16,46}$ in the unimolecular fragmentation processes, namely the evaporation of a monomer

$$
\mathrm{Na}_{n} \rightarrow \mathrm{Na}_{n-1}+\mathrm{Na}
$$

and the evaporation of a dimer

$$
\mathrm{Na}_{n} \rightarrow \mathrm{Na}_{n-2}+\mathrm{Na}_{2} \text {. }
$$

The same predominant channels are relevant for the fragmentation of cationic clusters and have been observed experimentally. ${ }^{47}$ Figure 7 shows the fragmentation energies corresponding to the evaporation of a monomer and a dimer, respectively, for $n \leq 14$,

$$
\begin{aligned}
& \Delta_{1}(n)=-E(n)+E(n-1)+E(1), \\
& \Delta_{2}(n)=-E(n)+E(n-2)+E(2) .
\end{aligned}
$$

These two quantities exhibit rather different behaviors. While $\Delta_{1}$ shows a very clear odd-even alternation, which reflects the odd-even alternation in the binding energies per atom, $\Delta_{2}$ does not present any periodicity at all. This results into a preference for even-numbered clusters to evaporate a monomer, while fragmentation into a dimer is the lowest fragmentation channel for odd-numbered

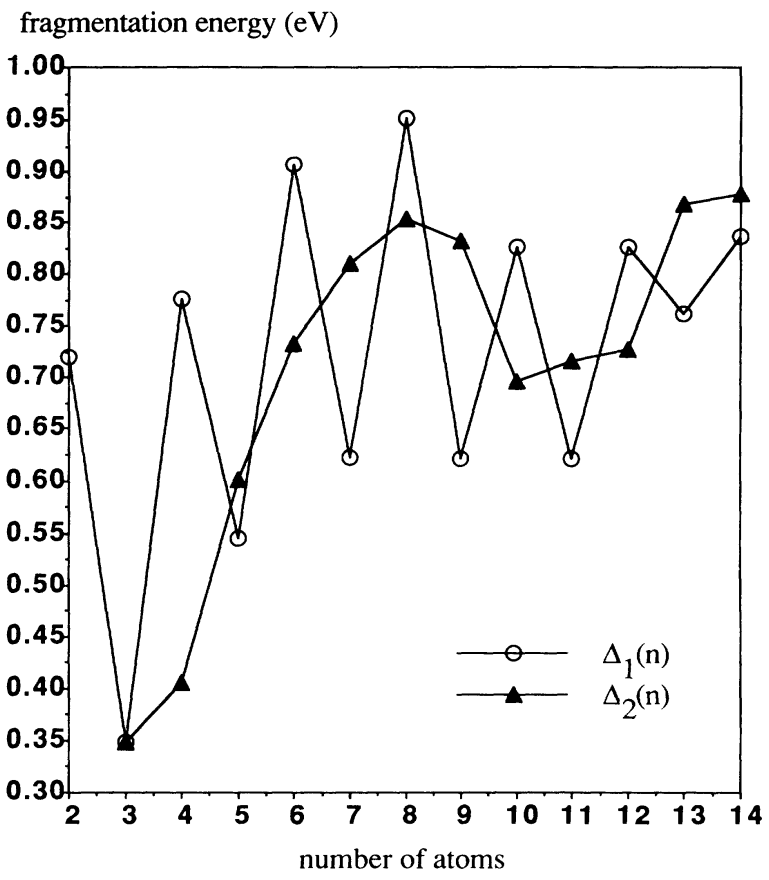

FIG. 7. Fragmentation energies of small sodium clusters into a monomer $\left[\Delta_{1}(n)\right]$ and a dimer $\left[\Delta_{2}(n)\right]$.

ones. The only exception is $n=14$. The dissociation energies (i.e., the fragmentation energies towards the lowest channel) are given in Table IV and compared to the results of other authors. As expected for magic numbers, $\mathrm{Na}_{2}$ and $\mathrm{Na}_{8}$ show stronger dissociation energies than their immediate neighbors. Those results are consistent with other previous theoretical calculations performed for the smallest clusters. ${ }^{11-16}$

\section{LARGE CLUSTERS AND EXTRAPOLATION TO THE BULK}

We examine in this section calculations of larger clusters involving up to a few hundredth of atoms, with specific geometries, namely regular Mackay icosahedra ${ }^{58}$ and regular cuboctahedra (pieces of fcc-like lattice) for $n=55,147,309$, and 561 and also cubes (pieces of bcclike lattice for $n=35,91,189,341$, and 559 corresponding to clusters with $2 \times 2 \times 2,3 \times 3 \times 3,4 \times 4 \times 4,5 \times 5 \times 5$, and $6 \times 6 \times 6$ primitive cells. These shapes may not correspond to the actual shapes of free clusters, however such calculations are likely to provide some information with regard to the convergency of cluster properties towards those in the bulk situation. Moreover, in a recent publication, Martin, Buttet, and Car report observations which might correspond to the completion of cuboctahedral or icosahedral shells of atoms for large clusters beyond $n=1500$ atoms, whereas stabilities of smaller clusters are better characterized by the filling of electronic shells. Thus, the examination of such structures may be of interest, even although we do not consider in the present work the range of interest $(n \geq 1500)$ implied in the work of Martin et al. ${ }^{59}$ For those structures, there is only one independent geometrical parameter, that has 
TABLE IV. Dissociation energies of $\mathrm{Na}_{n}$ clusters ( $\mathrm{De}=\operatorname{Min}\left[\Delta_{1}(n), \Delta_{2}(n)\right]$ in eV). densityfunctional theory, Martin, Car, and Buttet (Refs. 18 and 19) [DFT(MCB)]; density-functional theory, Röthlisberger and Andreoni (Ref. 29) [DFT (RA)]; all electron CI, Bonačić-Koutecký, Fantucci, and Koutecký (Ref. 14) (AE-CI); effective core potential CI, Bonačić-Koutecký, Fantucci, and Koutecký [ECP-CI (BFK)]; Spiegelmann and Pavolini (Ref. 16) [ECP-CI (SP)]; topological Hückel model, Wang et al. (Refs. 21 and 22) (HMO). The asterisk denotes fragmentation into a dimer.

\begin{tabular}{|c|c|c|c|c|c|c|c|c|}
\hline$n$ & DFT (MCB) & DFT(RA) & AE-CI & ECP-CI (BFK) & ECP-CI (SP) & HMO & This work & $\begin{array}{c}\text { Other } \\
\text { authors }\end{array}$ \\
\hline 2 & 0.90 & & 0.54 & 0.54 & 0.70 & 0.76 & 0.720 & $0.74^{\mathrm{a}}$ \\
\hline 3 & 0.39 & & 0.23 & 0.21 & 0.25 & 0.00 & 0.349 & $0.330^{\mathrm{b}}$ \\
\hline 4 & $0.64^{*}$ & $0.47^{*}$ & $0.45^{*}$ & $0.30^{*}$ & $0.36^{*}$ & $0.43^{*}$ & $0.405^{*}$ & $0.408^{c}$ \\
\hline 5 & 0.76 & 0.70 & 0.54 & 0.42 & 0.59 & 0.58 & 0.545 & \\
\hline 6 & $1.04^{*}$ & $1.00^{*}$ & $0.76^{*}$ & $0.62^{*}$ & $0.83^{*}$ & $0.85^{*}$ & $0.733^{*}$ & \\
\hline 7 & 1.36 & 0.97 & 0.51 & 0.49 & & 0.66 & 0.622 & \\
\hline 8 & 1.14 & 1.17 & $0.79^{*}$ & $0.65^{*}$ & & $1.06^{*}$ & $0.853^{*}$ & \\
\hline 9 & & 0.52 & 0.03 & & & 0.57 & 0.621 & \\
\hline 10 & & $0.73^{*}$ & & & & $0.57^{*}$ & $0.696^{*}$ & \\
\hline 11 & & & & & & 0.60 & 0.621 & \\
\hline 12 & & & & & & $0.74^{*}$ & $0.727^{*}$ & \\
\hline 13 & & & & & & 0.91 & 0.762 & \\
\hline 14 & & & & & & 0.91 & 0.836 & \\
\hline
\end{tabular}

${ }^{\text {a}}$ Verma et al. (Ref. 37).

${ }^{b}$ Deduced from the experimental work of Hilpert (Ref. 55).

${ }^{\mathrm{c} B o n a c ̌ i c ́-K o u t e c k y ́, ~ F a n t u c c i, ~ a n d ~ K o u t e c k y ́ ~(R e f . ~ 25) . ~}$

been optimized (it corresponds to the expansion of the cluster).

It can be seen in Fig. 8 that icosahedral and cuboctahedral structures are more stable than cubic structures, which can be understood as minimizing the number of surface atoms with respect to the number of atoms in the volume. For $n=55$, the icosahedron is more stable than the cuboctahedron. However an inversion occurs, both structures are almost degenerate at $n=147$ and cuboctahedra happen to be more stable for $n=309$ and $561 .^{60}$ Results obtained with DFT formalism ${ }^{20}$ for cuboctahedra $n=55$ and 147 are also illustrated in Fig. 8. In analogy with what was obtained for the smaller clusters the DFT binding energies are larger than ours.

At very low temperature, sodium bulk is characterized by the fcc lattice, whereas at room temperature, the bcc lattice is more stable with a binding energy of $1.13 \mathrm{eV}$. It is seen that even for $n=559$ or 561 , the bulk value is not yet reached. This is not surprising since surface atoms still represent a large proportion of the total number of atoms. In order to get a reasonable estimation of the bulk energy corresponding to the present calculations, the total binding energy was separated into different contributions, namely

$$
n e=n_{v} e_{v}+n_{s} e_{s} \text {. }
$$

$e_{v}$ and $e_{s}$ represent, respectively, the binding energies of volume and surface atoms. $e_{v}$ may be considered as close to the bulk energy, while $e_{s}$ is the energy required to remove a surface atom. For icosahedra and cuboctahedra, the values $e_{v}$ and $e_{s}$ are determined from $n=309$ and 561, whereas for cubic structures, they are determined from $n=341$ and 559 (see Table V). The fcc structure, in line with the extrapolation of cuboctahedral clusters, is found to be the most stable with a binding energy per atom
$(1.06 \mathrm{eV})$ very close to the experimental value whereas the extrapolation of icosahedral clusters yields $1.04 \mathrm{eV}$ and the extrapolation of bcc-like cubic clusters is $1.05 \mathrm{eV}$. The extrapolated energies for surface atoms are 0.77 and $0.69 \mathrm{eV}$, respectively, smaller than the $0.89-\mathrm{eV}$ value generally admitted. ${ }^{59}$

\section{CONCLUSION}

We have developed in the present paper a distancedependent extension of the Hückel model, on the basis of

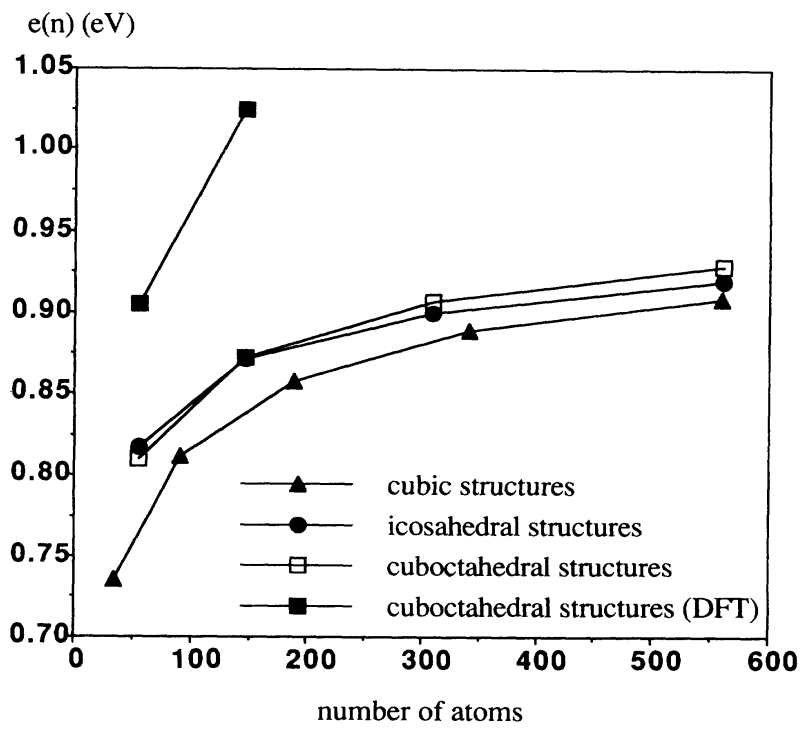

FIG. 8. Cohesive energies $\left(e_{v}\right)$ of large sodium clusters. The bulk value is $1.13 \mathrm{eV}$. Comparison is made with the DFT results of Martins (Ref. 20) for cuboctahedral clusters $n=55$ and 147. (Values are taken from the picture of Ref. 20.) 
TABLE V. Binding energy per atom (in eV) for large $\mathrm{Na}_{n}$ clusters.

\begin{tabular}{rccc}
\hline \multicolumn{1}{c}{$n$} & Cube & Icosahedra & Cuboctahedra \\
\hline 35 & 0.735 & & \\
55 & & 0.818 & 0.810 \\
91 & 0.812 & & \\
147 & & 0.871 & 0.872 \\
189 & 0.857 & & 0.907 \\
309 & & 0.900 & \\
341 & 0.886 & & 0.929 \\
559 & 0.909 & 0.920 & \\
561 & & & \\
\hline \hline
\end{tabular}

an effective Hamiltonian including the effects of $p$ orbitals in a perturbative way. This model allows for a quick determination of the PES. The use of Monte-Carlo simulated annealing together with this model potential-energy function yields for $n=3-9$ geometrical shapes of sodium clusters which are completely similar to CI $a b$ initio calculations following gradient SCF optimization. Binding energies have been shown to present a correct dependence as well for small clusters $(n<20)$ as for larger clusters with a few hundredths of atoms, and extrapolation to the bulk also seems to be consistent, at least from an energetic point of view. For small clusters in the range $n=10-19$, some relevant structures are proposed also obtained from Monte Carlo simulated annealing. Our results are fully consistent with the very recent work of Röthlisberger and Andreoni ${ }^{29}$ obtained with the DFT formalism and the Parinello-Car method. From the present results, it does not seem that any unique construction rule governs the genealogy and growth of alkali-metal clusters in the range $n=1-14$. However, some small clusters appear as cores, or pieces of larger ones. A frequent constituent occurs to be the pentagonal bipyramid $\left(\mathrm{Na}_{7}\right)$. However, some other cores can also be identified, such as the fully capped tetrahedron $\left(\mathrm{Na}_{8}\right)$. As a characteristic example, both substructures can be identified in the $\mathrm{Na}_{9}$ ground state. As other substructures, one can mention the bicapped square antiprism (the second structure of $\mathrm{Na}_{10}$ ) which appears in $\mathrm{Na}_{12}$ and one of the $\mathrm{Na}_{14}$ isomers. With regard to clusters beyond $\mathrm{Na}_{14}$, it is interesting to observe that $\mathrm{Na}_{15}, \mathrm{Na}_{16}$, and $\mathrm{Na}_{17}$ are pieces of the $\mathrm{Na}_{19}$ double icosahedron. The extensive and systematic research of all lowest isomers in the range $\mathrm{Na}_{15}-\mathrm{Na}_{21}$ is in progress, and may allow a better understanding of the rules concerning the growth of clusters for $n \geq 15$. However, the number of possible degenerate isomers with a rather low energy becomes crucially increasing, and it is very likely that thermodynamical or molecular-dynamics simulations should be more adequate for determining observed properties than the obtention of a few unique structures, depending of the experimental conditions obtained in experiments. Such treatments are feasible, at least for small clusters with the present model. In principle, the study of association and fragmentation processes from a dynamical point of view can also be undertaken, provided that a modification of the orbital filling rule and electrostatic corrections should be made in order to ensure correct dissociation for atoms with unpaired electrons. Such work is in progress.

\section{ACKNOWLEDGMENT}

The Laboratoire de Physique Quantique is unité associée No. 505 au Centre National de la Recherche Scientifique.
${ }^{1}$ A. L. Companion, Chem. Phys. Lett. 56, 500 (1978).

${ }^{2}$ W. D. Knight, K. Clemenger, W. A. de Heer, W. A. Saunders, M. Chou, and M. L. Cohen, Phys. Rev. Lett. 52, 2141 (1984).

${ }^{3}$ W. Ekardt, Phys. Rev. Lett. 52, 1925 (1984).

${ }^{4}$ K. Clemenger, Phys. Rev. B 32, 1359 (1985); 86, 619 (1986).

${ }^{5}$ M. L. Cohen, M. Y. Chou, W. D. Knight, and W. A. de Heer, J. Phys. Chem. 91, 3141 (1987).

${ }^{6}$ Y. Ishii, S. Ohnishi, and S. Sugano, Phys. Rev. B 31, 1804 (1985).

${ }^{7}$ K. Selby, M. Vollmer, J. Masui, V. Kresin, W. A. de Heer, and W. D. Knight, Phys. Rev. B 40, 5417 (1989).

${ }^{8}$ W. Ekardt and Z. Penzar, Phys. Rev. B 38, 4273 (1988).

${ }^{9}$ W. A. Saunders, Phys. Rev. B 40, 1400 (1989).

${ }^{10}$ S. Saito and M. L. Cohen, Phys. Rev. B 38, 1124 (1988).

${ }^{11}$ I. Boustani, W. Pewestorf, P. Fantucci, V. Bonačić-Koutecký, and J. Koutecký, Phys. Rev. B 35, 9437 (1987).

${ }^{12}$ I. Boustani and J. Koutecký, J. Chem. Phys. 88, 5652 (1988).

${ }^{13}$ V. Bonačić-Koutecký, I. Boustani, M. Guest, and J. Koutecký, J. Chem. Phys. 89, 4861 (1988).

${ }^{14}$ V. Bonačić-Koutecký, P. Fantucci, and J. Koutecký, Phys. Rev. B 37, 4369 (1988).

${ }^{15} \mathbf{P}$. Fantucci and J. Koutecký, in Elemental and Molecular Clusters, edited by G. Benedek, T. P. Martin, and G. Pac- chioni (Springer-Verlag, Berlin, 1988), p. 125.

${ }^{16}$ F. Spiegelmann and D. Pavolini, J. Chem. Phys. 89, 4954 (1988).

${ }^{17}$ J. Flad, G. Igel, M. Dolg, H. Stoll, and H. Preuss, Chem. Phys. 75, 331 (1983).

${ }^{18}$ J. L. Martins, R. Car, and J. Buttet, J. Chem. Phys. 78, 5646 (1983).

19J. L. Martins, J. Buttet, and R. Car, Phys. Rev. B 31, 1804 (1985).

20J. L. Martins, Z. Phys. D 12, 347 (1989).

${ }^{21}$ Y. Wang, T. F. George, D. M. Lindsay, and A. C. Beri, J. Chem. Phys. 86, 3493 (1987).

${ }^{22}$ D. M. Lindsay, Y. Wang, and T. F. George, J. Chem. Phys. 86, 3500 (1987).

${ }^{23}$ D. M. Lindsay, L. Chu, Y. Wang, and T. F. George, J. Chem. Phys. 87, 1685 (1987).

${ }^{24}$ V. Bonačić-Koutecký, P. Fantucci, and J. Koutecký, Chem. Phys. Lett. 146, 518 (1988).

${ }^{25}$ V. Bonačić-Koutecký, P. Fantucci, and J. Koutecký, Chem. Phys. Lett. 166, 32 (1990).

${ }^{26}$ V. Bonačic-Koutecký, M. M. Kappes, P. Fantucci, and J. Koutecký, Chem. Phys. Lett. 170, 26 (1990).

${ }^{27}$ R. Car and M. Parinello, Phys. Rev. Lett. 55, 2471 (1985). 
${ }^{28}$ P. Ballone, W. Andreoni, R. Car, and M. Parinello, Phys. Rev. Lett. 60, 271 (1988); W. Andreoni in Elemental and Molecular Clusters, edited by G. Benedek, T. P. Martins, and G. Pachioni (Springer-Verlag, Berlin, 1988), p. 206.

${ }^{29}$ U. Röthlisberger and W. Andreoni, J. Chem. Phys. 94, 8129 (1991).

${ }^{30}$ I. Stich, R. Car, and M. Parinello, Phys. Rev. B 39, 4997 (1989).

${ }^{31}$ F. Spiegelmann, P. Blaise, D. Maynau, and J. P. Malrieu, Z. Phys. D 12, 341 (1989).

${ }^{32}$ P. Blaise, F. Spiegelmann, D. Maynau, and J. P. Malrieu, Phys. Rev. B 41, 5566 (1990).

${ }^{33}$ J. Hubbard, Proc. R. Soc. London Ser. A 276, 238 (1963).

${ }^{34}$ E. Hückel, Z. Phys. 70, 204 (1931); 72, 320 (1931); 76, 628 (1932); 83, 632 (1933); R. Hoffman, J. Chem. Phys. 39, 1397 (1963).

${ }^{35}$ C. Bloch, Nucl. Phys. 6, 329 (1958); J. de Cloizeaux, Nucl. Phys. 20, 321 (1960); B. H. Brandow, Rev. Mod. Phys. 39, 771 (1967); Adv. Quantum Chem. 10, 187 (1977); Int. J. Quantum Chem. 15, 207 (1979); I. Lindgren and J. Morisson, Atomic Many Body Theory (Springer-Verlag, Berlin, 1982); F. Spiegelmann and J. P. Malrieu, J. Phys. B 17, 1259 (1984); J. P. Daudey and J. P. Malrieu, in Current Aspects of Quantum Chemistry, edited by R. Carbo (Elsevier, Amsterdam, 1982).

${ }^{36}$ G. Jeung, J. Phys. B 16, 4289 (1983).

${ }^{37}$ K. K. Verma, J. T. Bahns, A. R. Rejei-Rizi, W. C. Stwalley, and W. T. Zemke, J. Chem. Phys. 78, 3599 (1983).

${ }^{38}$ D. J. Chadi and R. M. Martin, Solid State Commun. 19, 643 (1976), Phys. Rev. Lett. 41, 1062 (1978); D. J. Chadi, Phys. Rev. B 29, 785 (1984).

${ }^{39}$ W. A. Harrison, Electronic Structure and the Properties of Solids (Freeman, San Francisco, 1980); O. F. Sankey and R. E. Allen, Phys. Rev. B 33, 7164 (1986); O. L. Alerhand and E. J. Mele, ibid. 35, 5533 (1987); 37, 2536 (1988); A. T. Paxton and A. P. Sutton, J. Phys. C 21, 481 (1988); R. Biswas, C. Z. Wang, C. T. Chan, K. M. Ho, and C. M. Soukoulis, Phys. Rev. Lett. 63, 1491 (1989); C. Z. Wang, C. T. Chan, and K. M. Ho, Phys. Rev. B 42, 11276 (1990); C. H. Xu, C. Z. Wang, C. T. Chan, and K. M. Ho, ibid. 43, 5024 (1991); C. Z. Wang, C. T. Chan and K. M. Ho, Phys. Rev. Lett. 66, 189 (1991).
${ }^{40}$ D. Tomanek and M. H. Schlüter, Phys. Rev. Lett. 56, 1055 (1986); Phys. Rev. B 36, 1208 (1987).

${ }^{41}$ A. P. Sutton, M. W. Finnis, D. G. Pettifor, and Y. Ohta, J. Phys. C 21, 35 (1988).

${ }^{42}$ J. A. Majewski and P. Vogl, Phys. Rev. B 35, 9666 (1987).

${ }^{43}$ M. Kohyama, R. Yamamoto, Y. Ebata, and M. Kinoshita, Phys. Status Solidi B 152, 533 (1989).

${ }^{44}$ S. Kirkpatrick, C. D. Gelatt, and M. P. Vecchi, Science 220, 671 (1983); S. Kirkpatrick, J. Stat. Phys. 34, 975 (1984).

${ }^{45}$ N. Metropolis, A. Rosenbluth, M. N. Rosenbluth, A. Teller, and E. Teller, J. Chem. Phys. 21, 1087 (1953).

${ }^{46}$ B. Rao and P. K. Jena, Phys. Rev. B 32, 2058 (1985); B. Jena, B. K. Rao, and R. H. Niemienen, Solid State Commun. 59, 509 (1986); B. K. Rao, P. Jena, M. Manninen, and R. M. Niemienen, Phys. Rev. Lett. 58, 1188 (1987).

${ }^{47}$ C. Bréchignac, Ph. Cahuzac, D. Pavolini, J. Ph. Roux, and F. Spiegelmann, J. Chem. Phys. 87, 5694 (1987).

${ }^{48}$ R. L. Martin and E. R. Davidson, Mol. Phys. 35, 1713 (1978).

${ }^{49}$ J. L. Martins, R. Car, and J. Buttet, J. Chem. Phys. 78, 5646 (1983).

${ }^{50}$ F. Cocchini, T. H. Upton, and W. Andreoni, J. Chem. Phys. 88, 6068 (1988).

${ }^{51}$ A. Herrman, H. Hoffman, S. Leutwyler, E. Schumacher, and L. Woste, Chem. Phys. Lett. 62, 216 (1979).

${ }^{52}$ M. Broyer, G. Delacretaz, P. Labastie, R. L. Whetten, J. P. Wolf, and L. Wöste, Z. Phys. D 3, 131 (1986).

${ }^{53} \mathrm{Ph}$. Dugourd, J. Chevaleyre, M. Broyer, J. P. Wolf, and L. Wöste, Chem. Phys. Lett. 175, 555 (1990).

${ }^{54}$ G. H. Jeung, M. Broyer, and P. Labastie, Chem. Phys. Lett. 165, 494 (1990).

${ }^{55}$ H. K. Hilbert, Ber. Bunsenges Phys. Chem. 88, 263 (1984).

${ }^{56}$ D. M. Mingos and Z. Lin, Chem. Phys. 137, 15 (1989).

${ }^{57}$ Z. Lin, T. Slee, and D. M. P. Mingos, Chem. Phys. 142, 321 (1990).

${ }^{58}$ A. L. Mackay , Acta Crystallogr. 15, 916 (1962).

${ }^{59}$ T. P. Martin, T. Bergmann, H. Gählich, and T. Lange, Chem. Phys. Lett. 172, 209 (1990).

${ }^{60}$ N. W. Ashcroft and N. D. Mermin, Solid State Physics (Holt Saunders, Tokyo, 1976). 